\title{
Development of a High-Fidelity Robot-Assisted Kidney Transplant Simulation Platform Using Three-Dimensional Printing and Hydrogel Casting Technologies
}

\author{
Patrick Saba, BA, Elizabeth Belfast, BS, ${ }^{1}$ Rachel Melnyk, MS, ${ }^{1}$ Ankit Patel, MD, \\ Randeep Kashyap, MD, MPH, ${ }^{2}$ and Ahmed Ghazi, MD, FEBU, MHPE ${ }^{1}$
}

\begin{abstract}
Introduction and Objective: Despite the adoption of robotic donor nephrectomy, the steep learning curve of robotic recipient transplantation has hindered the implementation of a complete robot-assisted kidney transplantation (RAKT). We sought to develop a high-fidelity perfused full immersion nonbiohazardous platform for RAKT simulation training.

Methods: A three-dimensional (3D) computer-aided design (CAD) model consisting of a kidney, pelvicaliceal system, renal artery, and vein was created from a CT scan of a donor patient. 3D printed casts designed from the CAD model were injected with various polyvinyl alcohol hydrogel formulations to fabricate an anatomical kidney phantom and surrounding abdominal cavity. The process was repeated using a recipient's CT scan to create the recipient pelvic model containing a bony pelvis, pelvic musculature, iliac arteries and veins, and bladder. Donor and recipient models each contained structures to simulate the perfused vascular and ureterovesical anastomosis. A board-certified transplant surgeon completed a robotic training curriculum, including four RAKT simulation procedures, from procurement of the donor kidney to final retroperitonealization. Metrics from the simulations (e.g., arterial, venous, ureterovesical, and total anastomosis times) were recorded and compared with surgical times from published data.

Results: The average time for the nephrectomies was $67.33( \pm 31.58)$ minutes. The average total anastomosis time was $60.85( \pm 9.73)$ minutes with $20.37( \pm 3.87), 20.17( \pm 4)$ and $15.1( \pm 2.35)$ minutes for arterial, venous, and ureterovesical anastomosis, respectively. The recorded arterial and venous anastomosis times were within published times for competency ( $\Delta=2.47$ and $\Delta=2.87$, respectively), whereas the uterovesical time was within the mastery range $(\Delta=0.45)$.

Conclusions: Using a combination of 3D printing and hydrogel casting technologies, a high fidelity perfused full-immersion nonbiohazardous simulation platform for RAKT was developed. The utilization of this platform has the potential to replace the early cases in a learning curve while decreasing the barriers to utilization for transitioning transplant surgeons.
\end{abstract}

Keywords: simulation, 3D printed, kidney transplant, robotic surgery

\section{Introduction}

$\mathbf{K}$ IDNEY TRANSPLANTATION (KT) IS an effective renal replacement therapy that reduces mortality rate and improves the quality of life of patients with end-stage renal disease. ${ }^{1}$ Open approaches are considered the standard for KT, ${ }^{2}$ but there has been a large push for adoption of minimally invasive approaches given their comparable outcomes and enhanced patient recovery. ${ }^{3,4}$ Laparoscopic techniques were once the epitome of these "minimally invasive" procedures; however, recent improvements in stereotactic vision, $10 \times$ magnification, instrument precision, and ergonomic efficiency have brought surgical robots to the forefront. ${ }^{5}$

Consequently, robot-assisted kidney transplantation (RAKT) for both donor and recipient procedures is gaining popularity with promising results in Centers of Excellence. ${ }^{6,7}$ RAKT claims smaller incisions, less wound-related complications (especially hernia, dehiscence, and infection), and faster recovery.

\footnotetext{
${ }^{1}$ Simulation Innovation Laboratory, Department of Urology, ${ }^{2}$ Department of Surgery, Transplant, University of Rochester Medical Center, Rochester, New York, USA.
} 
Despite these potential advantages, prolonged operation and ischemic time (cold and warm) must be considered. ${ }^{8} \mathrm{~A}$ recent publication reported that a minimum of 35 RAKT cases are necessary to reach reproducibility in terms of renal rewarming time (RWT), complications, and functional results. ${ }^{9}$ Ultimately, this steep learning curve may lead to lower graft and patient survival rates, which can hinder the integration and success of RAKT for recipients.

Current robotic simulators have demonstrated their usefulness as effective training tools for robotic surgery. ${ }^{10}$ However, these expensive tools remain limited to the initial phase of training; significant progress is needed to address advanced skill acquisition. ${ }^{11}$ Cadaveric and animal models remain the gold standard for realistic procedural simulation, but their high cost, regulated availability, risks of transferable diseases, and potential ethical concerns limit their use. ${ }^{12} \mathrm{We}$ sought to overcome these limitations by developing a highfidelity perfused full-immersion nonbiohazardous RAKT training simulator consisting of both donor and recipient procedures by combining three-dimensional (3D) printing and hydrogel casting technologies.

\section{Methods}

\section{Fabrication of the simulation model}

The simulation platform is composed of two separate, but interconnected, units: a donor nephrectomy model and a recipient pelvic cavity. For the donor model, Digital Imaging and Communications in Medicine (DICOM) data from the CT scan of a patient scheduled for a living kidney donor procedure was segmented into a 3D computer-aided design (CAD) model using Mimics software (Materialize, Leuven, Belgium) (Fig. 1). The 3D CAD model included the kidney parenchyma with a hollow pelvicaliceal system, abdominal aorta, renal artery, inferior vena cava, and renal vein (Fig. 1).

The CAD files were then used to design injection casts of the desired anatomy, which were printed using a Fusion3 F400-S 3D printer (Fusion3 Design, Greensboro, NC) and then injected with a polyvinyl alcohol (PVA) hydrogel mixture. The resulting synthetic tissue can replicate various tissue types and their respective visual and tactile properties.

To build both the donor and recipient models, $\$ 95$ of materials and eight working hours are required. This process, created by the Simulation Innovation Lab (University of Rochester Medical Center, Rochester, NY), was effectively utilized to create a perfused kidney phantom for partial nephrectomy simulations that mimics the mechanical and functional properties of all components of fresh kidney tissue. ${ }^{13}$

The result of the application of this process is a perfused hydrogel donor kidney graft model that allows the surgeon to mimic dissection, cauterization, and suturing (Fig. 1). To finalize the construction of the donor nephrectomy model, the kidney phantom and other PVA structures (retroperitoneal fat and muscle, colon segment, spleen, and anterior abdominal wall) are assembled in an anatomic orientation and subjected
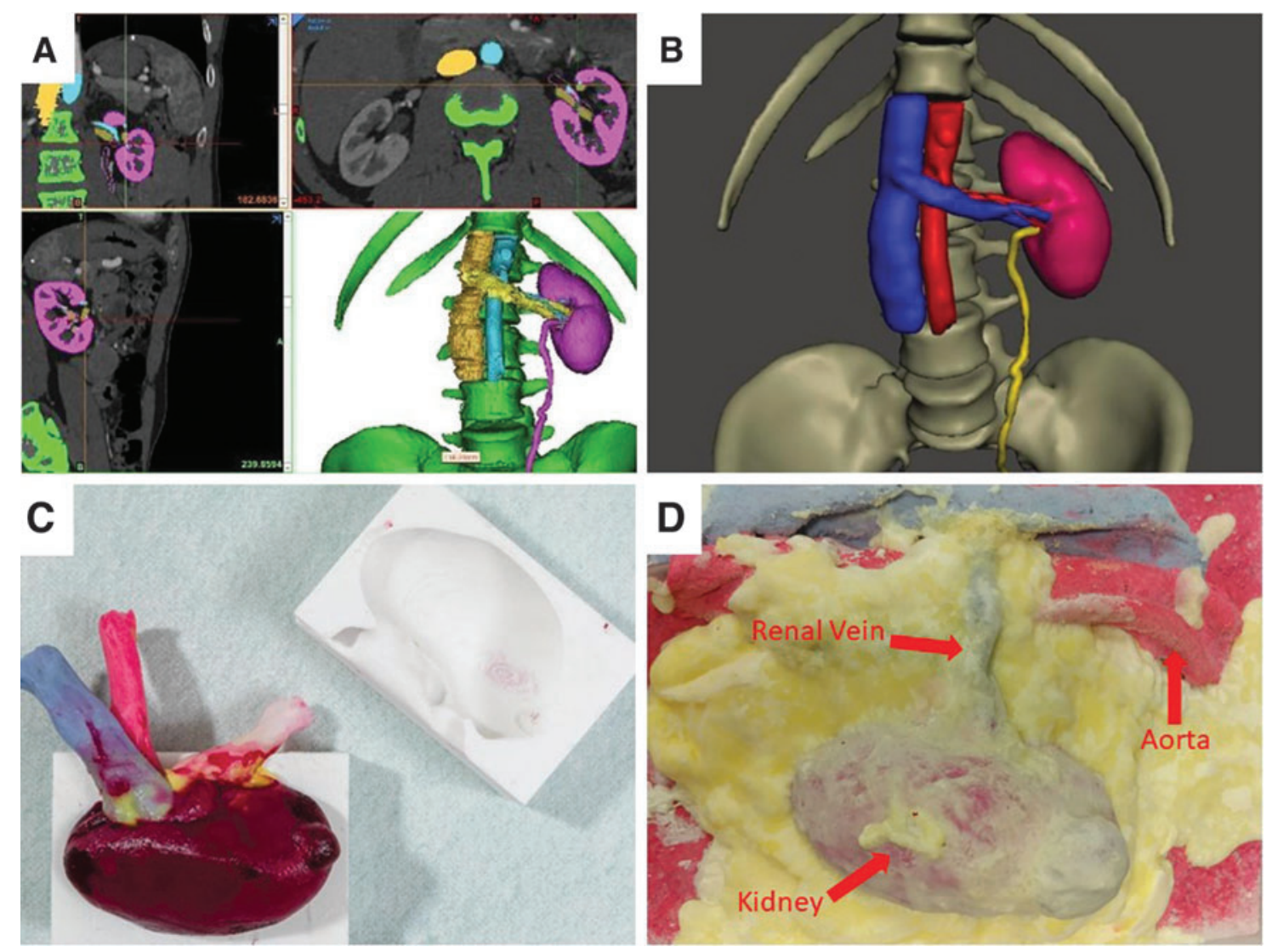

FIG. 1. Segmentation of CT scans using Mimics segmentation software and development of 3D CAD models for donor nephrectomy hydrogel model. (A) Mimics segmentation, (B) CAD model, (C) hydrogel donor kidney, (D) donor hydrogel nephrectomy model. $3 \mathrm{D}=$ three-dimensional; $\mathrm{CAD}=$ computer-aided design. 
FIG. 2. Robotic donor nephrectomy hydrogel simulation. (A) Stapling the renal artery, (B) stapling the renal vein.
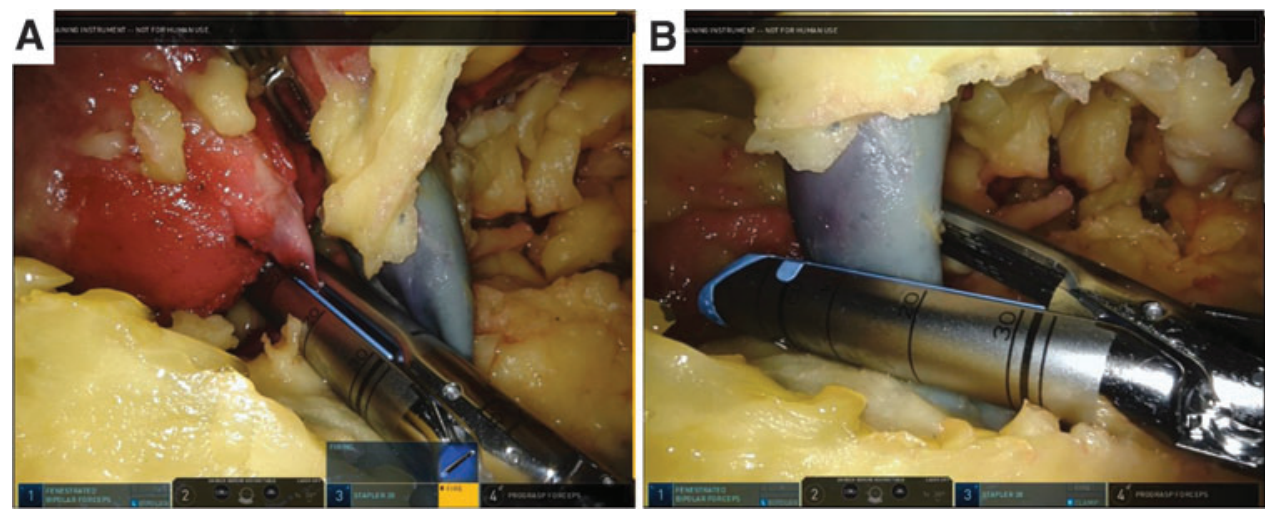

to final processing for organ cohesion (Fig. 1). Artificial blood is perfused through the kidney vasculature to simulate bleeding. The finished donor nephrectomy model assembly allows for all necessary steps, including the isolation and ligation of the renal vessels (Fig. 2).

A similar process was utilized to fabricate the recipient pelvic cavity model. The $\mathrm{CT}$ scan of the KT recipient was used to identify and segment the bony pelvis, bladder, right iliac arteries, and veins to create a recipient CAD model (Fig. 3). The CAD model was utilized to 3D print the pelvis and injection casts to fabricate a water-tight bladder and hollow iliac vessels. To replicate the ureterovesical anastomosis, the bladder was constructed to contain multiple tissue layers (mucosal, muscular, and serosal) and stratified in the pelvis to recreate the pelvic cavity anatomy (Fig. 3).

Mechanical testing following an established protocol was performed to determine the Young's modulus of human iliac vessel segments discarded from liver transplant surgeries. ${ }^{13}$ The same test was done on a catalogue of various PVA conditions (changing freeze thaws, concentrations, and additives) until an acceptable replicate was found that mimicked the Young's modulus value of the live tissue (average Young's modulus $=28.125 \mathrm{MPa}$ ).

Each assembled simulation model is placed into a da Vinci abdominal trainer box and docked to the da Vinci surgical robot to complete all the necessary steps of the recipient transplantation operation, including peritoneal
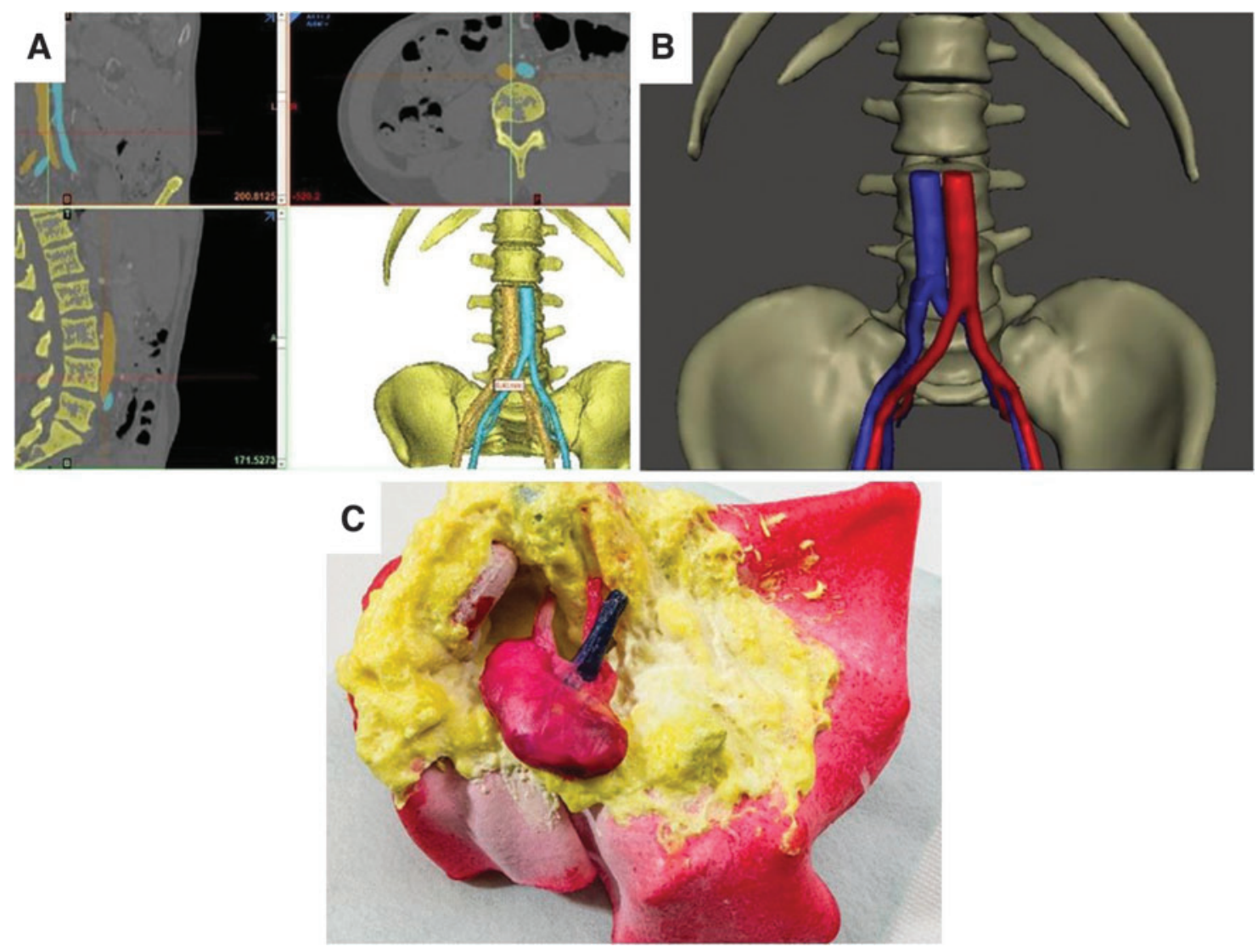

FIG. 3. Segmentation of CT scans using Mimics segmentation software and development of 3D CAD models for recipient hydrogel abdominal model. (A) Mimics segmentation, (B) recipient CAD model, (C) hydrogel pelvic recipient model preoperative. 
reflection and dissection, exposure of the bladder dome, regional hypothermia, positioning of the kidney, and finally, the arterial, venous, and ureterovesical anastomosis (with placement of J-stent) (Fig. 4).

\section{Surgeon training}

A board-certified kidney transplant surgeon from the University of Rochester Medical Center with an interest in transitioning to RAKT volunteered to participate in the simulations. The surgeon has 20 years of surgical transplant experience with an annual caseload of 75-100 transplants $(\sim 1500$ career transplants) and completed $>100$ robotic general surgery cases before this study, establishing foundational knowledge for the robotic approach. The surgeon completed a three-stage protocol.

(1) Familiarization with the RAKT technique: The surgeon completed an observership at a high-volume center under supervision of an expert in the field of RAKT. ${ }^{14}$

(2) Completion of da Vinci surgery online ${ }^{15}$ training pathway: Containing online modules, virtual reality simulation, and hands-on drills practicum that achieved familiarity with setup during robotic surgery, trocar placement, docking, as well as proficiency in basic skills essential for robotic surgery.

(3) Full immersion simulations: Utilizing these high-fidelity simulations in a mock operating room, including auxiliary personnel (bedside assist and circulator), the surgeon participated in four simulated procedures for a 2-month timeframe. Every simulation consisted of an abdominal donor model and recipient pelvic model, each designed from pairs of previous de-identified donor and recipient matched transplant patients. Each simulation was recorded for a postsimulation debrief that included self-reflection and review of technical aspects.

Setup for the simulation required $\sim 10$ minutes for the donor model (positioning the model into a da Vinci abdominal trainer and docking the robot) and $\sim 10$ minutes to swap the donor model with the recipient model. The surgeon first performed a total nephrectomy, isolating and ligating the renal artery and vein using a robotic stapler (Fig. 2). The donor kidney graft was extracted and wrapped in gauze to simulate the start of cold ischemia time. The robot was then undocked, and the donor abdominal model was switched with the pelvic recipient model. The time required to switch models was removed from the cold ischemia time and total operative time.

After docking, the recipient portion began with the surgeon reflecting the peritoneum and isolating the iliac vessels. Upon the surgeon's request, the donor kidney was introduced into the pelvic cavity through a side opening and placed in the optimal position for anastomosis. Excess vessel length was tailored, and vascular control was obtained using a pair of bulldog clamps on the renal vein.

Venous and arterial anastomosis times were measured from the first suture throw to the final knot (Fig. 4) after which blood flow was re-established (Fig. 4). The ureterovesical anastomosis was completed between the mucosa of the ureter and bladder and measured from the first needle throw to the final knot placement (Fig. 4). All suturing was completed using Black Diamond microforceps and 6-0 Gore Tex suture.

The simulation concluded when all three anastomoses were complete, and the kidney was reflected into the abdominal peritoneum allowing for kidney perfusion and
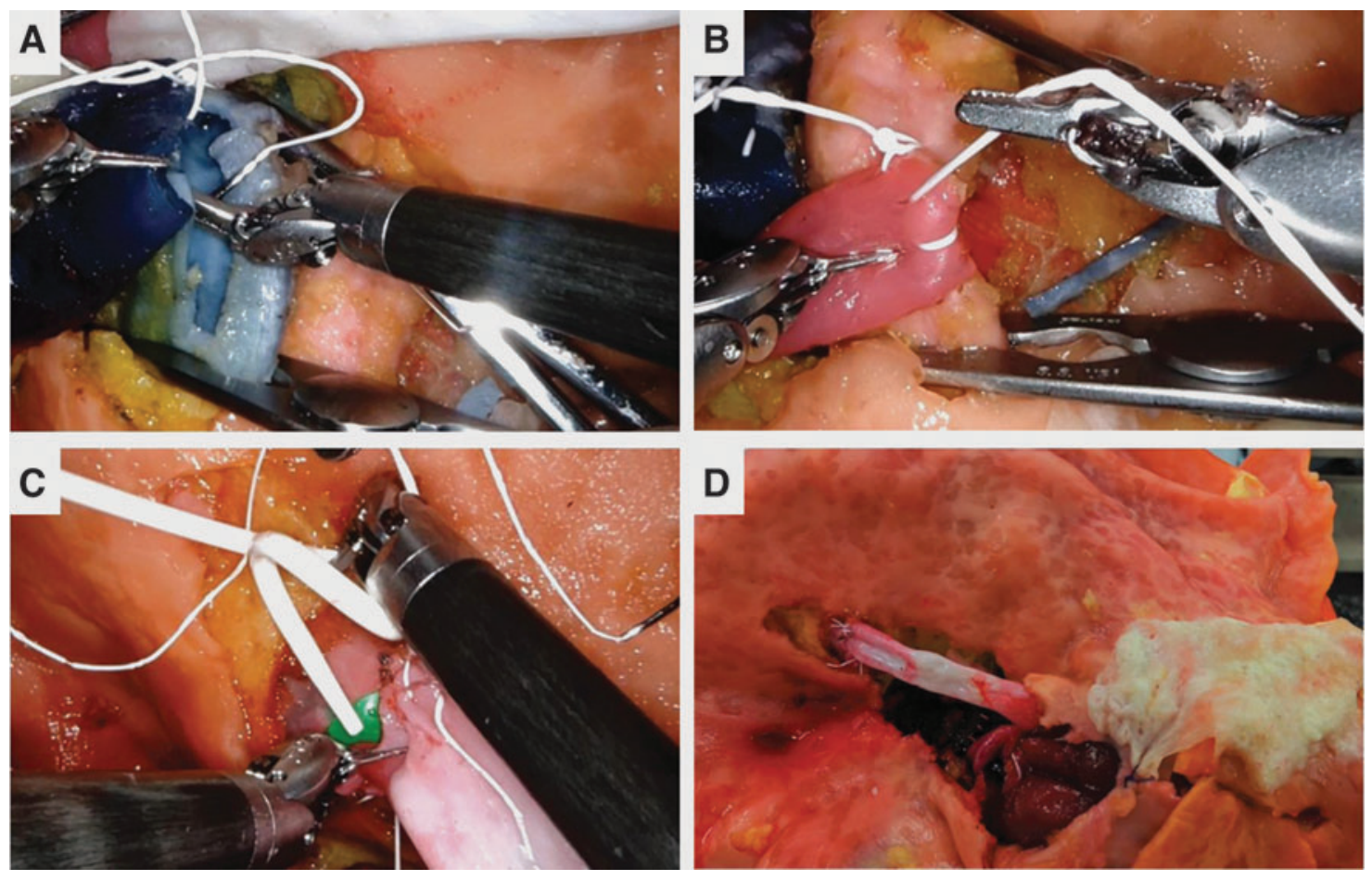

FIG. 4. Hydrogel recipient simulation. (A) Venous anastomosis, (B) arterial anastomosis, (C) ureterovesical anastomosis with insertion of a J stent, (D) hydrogel pelvic recipient model after completion of the anastomosis and retroperitonealization of the graft. 
Table 1. Surgical Metrics Comparison from Simulations vs Ahlawat Et Al. (Literature)

\begin{tabular}{lcccrr}
\hline Times (minutes) & $\begin{array}{c}\text { Simulation } \\
\text { average }\end{array}$ & $\begin{array}{c}\text { Literature } \\
\text { competency }\end{array}$ & $\begin{array}{c}\text { Literature } \\
\text { proficiency }\end{array}$ & $\begin{array}{c}\text { Literature } \\
\text { mastery }\end{array}$ & $\begin{array}{c}\Delta \text { minutes }= \\
\text { Simulation }- \text { Competency }\end{array}$ \\
\hline Arterial anastomosis time & $20.37 \pm 3.87$ & $17.9 \pm 2.79$ & $15.1 \pm 2.79$ & $12.4 \pm 2.79$ & 2.47 \\
Venous anastomosis time & $20.17 \pm 4$ & $17.3 \pm 2.13$ & $15.1 \pm 2.13$ & $13 \pm 2.13$ & 2.87 \\
Ureterovesical anastomosis time & $15.1 \pm 2.35$ & $25.2 \pm 4.44$ & $20.7 \pm 4.44$ & $16.3 \pm 4.44$ & -10.10 \\
Total anastomosis time & $60.85 \pm 9.73$ & $60.4 \pm 8.65$ & $50.9 \pm 8.65$ & $41.7 \pm 8.65$ & 0.45 \\
\hline
\end{tabular}

filtration into the bladder (Fig. 4). Anastomotic times (arterial, venous, and ureterovesical, along with total anastomosis time) were collected from video review. Total anastomosis time included vascular and ureterovesical anastomosis times and any required revisions. Each time point was compared with a published RAKT series with similar surgical techniques and surgeon background ${ }^{16}$ after a literature review of RAKT learning curves.

\section{Results}

The average time for the nephrectomies was $67.33( \pm 31.58)$ minutes; however, in one case a vascular injury to the renal vein occurred requiring homeostatic control. The repair was performed robotically without the need for conversion. Excluding repair time, the average nephrectomy time decreased to $62( \pm 24.04)$ minutes.

Mean arterial anastomosis time, venous anastomosis time, and ureterovesical anastomosis time were $20.37 \pm$ $3.87,20.17 \pm 4.0$, and $15.10 \pm 2.35$ minutes, respectively (Table 1). The mean total anastomosis time was $60.85 \pm 9.73$ minutes (Table 1).
The primary published series used for comparison defined the skill levels of competency (bare minimum skill required), proficiency (level of skill that is higher than the minimum), and mastery (extreme skill or command over the procedure) by a standard deviation of improvement in the respective anastomosis times. On average, competency was reported after completing 9 cases, proficiency after reaching 16 cases, and mastery after reaching 21 cases. ${ }^{16}$ In our study, anastomosis times were calculated using similar definitions as the literature (start of anastomosis to tying of the last suture).

Upon completion of the simulations, the surgeon was able to perform the arterial and venous anastomosis within the competency times $(17.9 \pm 2.79$ and $17.3 \pm 2.13$, respectively) reported in literature $(\Delta=2.47$ and $\Delta=2.87$, respectively) (Table 1). Interestingly, the surgeon completed the ureterovesical anastomosis with an average time 10 minutes faster than competency range, within the mastery range $(15.10 \pm 2.35$ vs $16.3 \pm 4.44$ minutes, $\Delta=-1.2)$. Although the different anastomoses fell in different skill levels, the average overall anastomosis time fell within the competency range of the reported literature $(60.85 \pm 9.73$ vs 60.4 minutes, $\Delta=0.45)$ (Table 1) (Fig. 5).

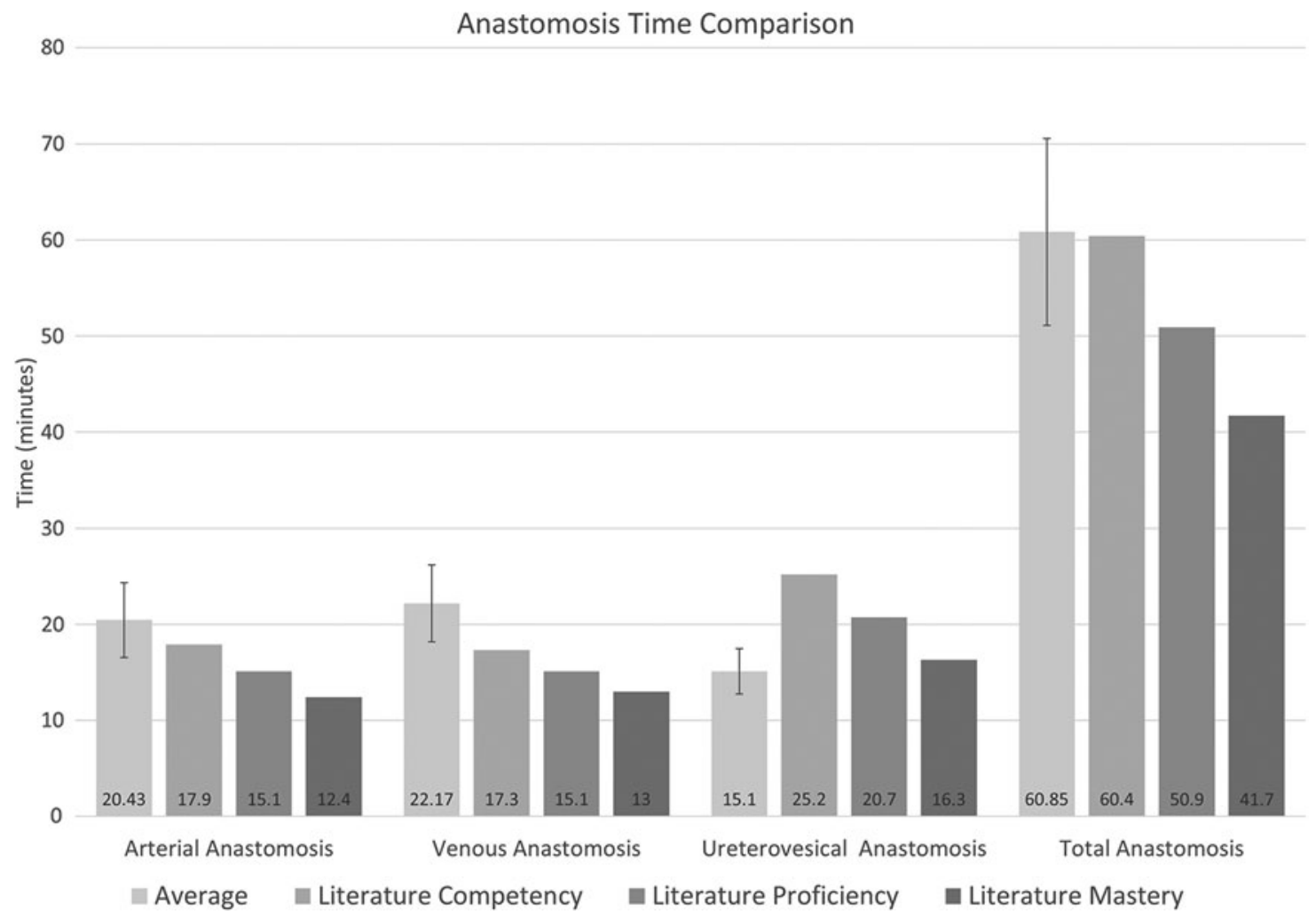

FIG. 5. Simulation vs Ahlawat et al. (literature) anastomosis time comparison graph with simulation standard deviation. 


\section{Discussion}

Using a combination of 3D printing and hydrogel casting, we created a high-fidelity simulation that mimics the tactile properties of live tissue and requires advanced procedural skills similar to that of live surgery. The perfusion capacity and realistic tissue texture, confirmed by a series of mechanical tests, provide a full immersion environment that is often missing in other simulations. The ability of the surgeon to obtain anastomosis times overlapping with published competency values indicates that during the simulated procedure, the surgeon encountered levels of difficulty similar to that of live cases.

The anastomosis time is one of the most important surgical metrics as it relates to the RWT of the kidney. RWT is defined as, "time between removal of the kidney from cold storage and the start of reperfusion while continuously adding ice slush," the bulk of which is taken up by the anastomoses. ${ }^{17}$ Marzouk et al. describe the clinical impact of longer anastomosis times regarding the increased likelihood of delayed graft functions (DGFs), prolonged length of postoperative hospital stay, and the potential increased use of hospital resources. ${ }^{18}$ This simulation platform addresses the need for improved anastomosis training (which can lead to a reduction in RWT and ultimately, reduction in DGF) as the main contributor to clinical outcomes.

Traditionally, surgical training outside of an operating room utilizes cadavers to supplement surgical training gaps and has been shown to improve surgical time metrics for KT. ${ }^{19}$ Huri et al. state that although fresh-frozen cadavers offer the optimal platform for laparoscopic surgical training because of their anatomical accuracy and tissue realism, their use is restricted by limited availability and a high financial cost. ${ }^{20}$ Cadavers also require storage, costly disposal, and either embalming techniques, freezer space, or other forms of biohazardous preservation. $^{20,21}$

Our nonbiohazardous high-fidelity anatomically accurate simulation platform can serve to bolster RAKT surgical education by offering similar advantages to cadavers (anatomic realism and live tissue fidelity) while also decreasing barriers to utilization (cost, availability, safety, and convenience). In addition, our fabrication process creates the potential for patientspecific rehearsals before live surgery by utilizing corresponding donor and recipient imaging. A standardized model can also be produced to reduce processing time (negating the need for multiple CAD designs and 3D printed casts), further decreasing costs and promoting reproducibility for widespread use.

The concept of creating a KT simulation model using 3D printing has previously been shown to help in preoperative planning as well as surgical training. ${ }^{22,23}$ Previous models boast similar benefits in terms of anatomical fidelity; however, many of these models lack true live tissue fidelity. ${ }^{23}$

Uwechue et al. demonstrated the value of a hybrid simulation model for RAKT by combining a 3D printed cradle (containing an anatomically accurate pelvis, iliac/renal vessel attachment supports, and kidney without vessels), with deceased donor vessels. Two robotic surgeons performed vascular anastomoses between the donor renal and iliac vessels that were splinted between the 3D printed vessel supports. The purpose of the model was to optimize the surgeons' training in vascular anastomoses in a reproducible manner to allow objective assessment of their competencies. ${ }^{22}$ However, by using cadaveric tissue, many of the same limitations seen in cadaver simulations remain, counteracting the benefits of 3D models. Our integration of the custom fabrication properties of 3D printing, along with live tissue tactile replication from the PVA hydrogel creates an optimized training platform.

The findings of our study must be understood in the context of the following limitations. This is a single surgeon single center trial limited to four simulations; it is expected that there was not a significant improvement in skill progression and that the final reported metrics remained within the surgeon's learning curve. An explanation may be that because of the four unique sets of donor and recipient CT scans that were utilized, which created variations in kidney size, location, angle, and shape of its vessels. Since a surgeon will never repeat the same live transplant, this variation prevented the surgeon from becoming familiar with the model allowing the surgeon to adapt overall surgical skills and not those specific to a single case.

In addition, although the primary referenced literature closely matches our study, the single-surgeon background limits the generalization of our results. Ultimately, determining the learning curve required to translate to competent RAKT performance would involve standardization of the model in the context of a multicenter multisurgeon study.

\section{Conclusion}

Using a combination of 3D printing and hydrogel casting technologies, we were able to create a high-fidelity perfused full-immersion nonbiohazardous simulation platform for RAKT procedures. Although simulation can never replace intraoperative experiences nor knowledge gained from experienced teachers, the integration of this platform has the potential to replace the early cases in the learning curve. Ultimately, decreasing barriers to utilization will increase training and will limit patient risk caused by inexperience. Further studies are warranted utilizing a larger sample size while incorporating varying teaching modalities and methods of assessment to evaluate the impact this simulation platform may have in optimizing the learning curve of developing surgeons.

\section{Author Disclosure Statement}

A.G. is consultant for Olympus and Johnson and Johnson. Research grant was from Intuitive. R.K. is consultant for Intuitive (All unrelated to this project). All other authors have no conflicts of interest.

\section{Funding Information}

This research did not receive any specific grant from funding agencies in the public, commercial, or not-for-profit sectors.

\section{References}

1. Abecassis M, Bartlett ST, Collins AJ, et al. Kidney transplantation as primary therapy for end-stage renal disease: A National Kidney Foundation/Kidney Disease Outcomes Quality Initiative (NKF/KDOQITM) conference. Clin J Am Soc Nephrol 2008;3:471-480.

2. Modi P, Pal B, Modi J, Kumar S, Sood A, and Menon M. Robotic assisted kidney transplantation. Indian J Urol 2014; 30:287-292.

3. Pein U, Girndt M, Markau S, et al. Minimally invasive robotic versus conventional open living donor kidney transplantation. World J Urol 2019;38:795-802. 
4. Tuğcu V, Şener NC, Şahin S, Yavuzsan AH, Akbay FG, Apaydin S. Robot-assisted kidney transplantation: Comparison of the first 40 cases of open vs robot-assisted transplantations by a single surgeon. BJU Int 2018;121:275-280.

5. Kumar R, Hemal AK. Emerging role of robotics in urology. J Minim Access Surg 2005;1:202-210.

6. Garcia-Roca R, Garcia-Aroz S, Tzvetanov I, Jeon H, Oberholzer J, Benedetti E. Single center experience with robotic kidney transplantation for recipients with BMI of $40 \mathrm{~kg} / \mathrm{m} 2$ or greater: A comparison with the UNOS registry. Transplantation 2017;101:191-196.

7. Abaza R, Ghani KR, Sood A, et al. Robotic kidney transplantation with intraoperative regional hypothermia: Robotic kidney transplantation. BJU Int 2014;113:679-681.

8. Wagenaar S, Nederhoed JH, Hoksbergen AWJ, Bonjer HJ, Wisselink W, van Ramshorst GH. Minimally invasive, laparoscopic, and robotic-assisted techniques versus open techniques for kidney transplant recipients: A systematic review. Eur Urol 2017;72:205-217.

9. Gallioli A, Territo A, Boissier R, et al. Learning curve in robot-assisted kidney transplantation: Results from the European Robotic Urological Society Working Group. Eur Urol 2020 [Epub ahead of print]; DOI: 10.1016/j.eururo .2019.12.008.

10. Abboudi H, Khan MS, Aboumarzouk O, Guru KA, Challacombe B, Dasgupta P, Ahmed K. Current status of validation for robotic surgery simulators-A systematic review. BJU Int 2013;111:194-205.

11. Ahmed K, Jawad M, Abboudi M, et al. Effectiveness of procedural simulation in urology: A systematic review. J Urol 2011;186:26-34.

12. Gilbody J, Prasthofer AW, Ho K, Costa ML. The use and effectiveness of cadaveric workshops in higher surgical training: A systematic review. Ann R Coll Surg Engl 2011; 93:347-352.

13. Melnyk R, Ezzat B, Belfast E, et al. Mechanical and functional validation of a perfused, robot-assisted partial nephrectomy simulation platform using a combination of $3 \mathrm{D}$ printing and hydrogel casting. World J Urol 2020;38:1631-1641.

14. Pahari H, Waldman B, Stracke J, et al. Robotic assisted live donor kidney transplantation-technique and outcomes. Am J Transplant 2018;18:379.

15. da Vinci Surgery Community. https://www.davincisurgery community.com/

16. Ahlawat RK, Tugcu V, Arora S, et al. Learning curves and timing of surgical trials: Robotic kidney transplantation with regional hypothermia. J Endourol 2018;32:116-1165.

17. Breda A, Territo A, Gausa L, et al. Robot-assisted kidney transplantation: The European experience. Eur Urol 2018; 73:273-281.
18. Marzouk K, Lawen J, Alwayn I, Kiberd BA. The impact of vascular anastomosis time on early kidney transplant outcomes. Transplant Res 2013;2:8.

19. Sutton ERH, Billeter A, Druen D, Roberts H, Rice J. Development of a human cadaver model for training in laparoscopic donor nephrectomy. Clin Transplant 2017; 31:e12979.

20. Huri E, Ezer M, Chan E. The novel laparoscopic training 3D model in urology with surgical anatomic remarks: Fresh-frozen cadaveric tissue. Turk J Urol 2016;42:224229.

21. Cabello R, González C, Quicios C, Bueno G, García JV, Arribas AB, Clascá F. An experimental model for training in renal transplantation surgery with human cadavers preserved using W. Thiel's embalming technique. J Surg Educ 2015;72:192-197.

22. Uwechue R, Gogalniceanu P, Kessaris N, et al. A novel 3D-printed hybrid simulation model for robotic-assisted kidney transplantation (RAKT). J Robot Surg 2018;12: 541-544.

23. Kusaka M, Sugimoto M, Fukami N, et al. Initial experience with a tailor-made simulation and navigation program using a 3-D printer model of kidney transplantation surgery. Transplant Proc 2015;47:596-599.

Address correspondence to: Ahmed Ghazi, MD, FEBU, MHPE Simulation Innovation Laboratory Department of Urology

University of Rochester Medical Center 601 Elmwood Avenue Rochester, NY 14642 USA

E-mail: ahmed_ghazi@URMC.rochester.edu

$\begin{aligned} & \quad \text { Abbreviations Used } \\ & 3 \mathrm{D}=\text { three dimensional } \\ & \mathrm{CAD}=\text { computer-aided design } \\ & \mathrm{CT}=\text { computed tomography } \\ & \mathrm{DGF}=\text { delayed graft function } \\ & \text { DICOM }=\text { digital imaging and communications in medicine } \\ & \mathrm{KT}=\text { kidney transplant } \\ & \mathrm{PVA}=\text { polyvinyl alcohol } \\ & \mathrm{RAKT}=\text { robot-assisted kidney transplant } \\ & \mathrm{RWT}=\text { rewarming time }\end{aligned}$

